\title{
Intervenciones para dejar de fumar en los centros de atención a las drogodependencias de Cataluña: La adicción abandonada
}

\section{Smoking cessation interventions in substance use disorders treatment centers of Catalonia: The abandoned addiction}

\author{
Gemma Nieva*, Montse Ballbì*****, Margarita Cano****, Blanca Carcolé*****, Teresa \\ Fernández****, Àngela Martínez******, Sílvia Mondon***, Antònia Raich*******, Pilar \\ Roig $* * * * * * * *$, Imma Serra*********, Jaume Serrano**********, Cristina Pinet***********.
}

\footnotetext{
* Unidad de Conductas Adictivas, Servicio de Psiquiatría. Hospital Universitario Vall d'Hebron; Vall d'Hebron Institut de Recerca (VHIR); Departamento de Psicología Clínica y de la Salud. Universidad Autónoma de Barcelona, Barcelona; Clínica Galatea, Barcelona.

** Unidad de Control del Tabaco, Instituto Catalan de Oncología. IDIBELL, Hospitalet de Ll.

*** Unidad de Conductas Adictivas, Departamento de Psiquiatría. Instituto de Neurociencias, Hospital Clínic, IDIBAPS, Barcelona. **** Centro de Atención a las Drogodependencias de Mataró, Servicio de Salud Mental y Adicciones.

Hospital de Mataró, Mataró.

***** Centro de Atención a las Drogodependencias de Tarragona, Tarragona.

****** Unidad de Tabaquismo, CAP Plana-Lledó, Vallès Oriental, Barcelona; Instituto Catalán de la Salud (ICS), Barcelona. ******* Grupo de Investigación SAMIS, Departamento de Salud Mental. Althaia Red Asistencial Universitaria de Manresa, Barcelona. ******** Centro de Atención a las Drogodependencias de Reus, Servicio de Salud Mental y Adicciones. Hospital de Reus, Tarragona. ********* Centro de Atención a las Drogodependencias de Sabadell, Sabadell.

*********** Centro de Atención a las Drogodependencias Horta-Guinardó, Barcelona.

*********** Unidad de Conductas Adictivas, Departamento de Psiquiatría. Hospital de la Santa Creu i Sant Pau, Barcelona.
}

\section{Resumen}

Los centros de tratamiento de drogodependencias son un recurso óptimo para realizar intervenciones para la cesación tabáquica (ICT). El objetivo de este estudio fue examinar la implementación de ICT en la Red de centros de Atención a las Drogodependencias (CAS) de Cataluña, así como evaluar las opiniones sobre la adecuación y viabilidad de la provisión de ICT. Los responsables de los CAS $(\mathrm{n}=57)$ contestaron un cuestionario on-line compuesto por 30 ítems. Se obtuvieron datos de 50 centros $(87,7 \%$ tasa de respuesta). El $46 \%$ de los CAS ofrecía algún tipo de ICT, pero sólo un 4,8\% de los nuevos pacientes eran tratados para dejar de fumar. Además, los responsables informaron que el 73,3\% de los profesionales que trabajaban en los CAS no había recibido formación en ICT. El $64 \%$ de los responsables estaba de acuerdo que todos los profesionales deberían realizar ICT. Aquellos centros que ofrecían ICT visitaban más pacientes y era más probable que tuviesen profesionales formados en ICT, comparado con los centros que no ofrecían ICT. La implementación de ICT en los CAS de Cataluña era subóptima. Se debería facilitar formación continuada a los profesionales de los CAS. No inter-

\begin{abstract}
Substance use disorders (SUD) treatment centers are an optimal setting for delivering smoking cessation interventions (SCI). This study aimed to examine the adoption of SCI in SUD treatment centers in Catalonia (Spain) as well as to assess their managers' views on the appropriateness and feasibility of providing SCI. Managers directly in charge of SUD treatment centers $(n=57)$ answered a 30 -item on-line questionnaire. Data was obtained of 50 centers $(87.7 \%$ response rate). Forty-six per cent of the centers provided some kind of SCI, but only $4.8 \%$ of the new patients were treated for smoking cessation. Managers reported that $73.3 \%$ of mental health professionals working in SUD centers had not been trained in SCI. Sixty-four per cent of managers agreed that all health professionals should deliver SCI. Those centers offering SCI attended more patients and were more likely to have professionals trained in SCI than those not offering SCI. The implementation of SCI in SUD treatment centers in Catalonia was suboptimal. Continuing education and training should be provided for all health professionals working in SUD centers. Not systematically
\end{abstract}


venir sobre el consumo de tabaco en pacientes en tratamiento por otras drogodependencias significa perder oportunidades para reducir costes en salud y económicos mientras perpetuamos una cultura fumadora. Palabras clave: Dejar de fumar; trastornos por consumo de sustancias; centros de tratamiento de drogodependencias; actitud del personal sanitario; formación. delivering SCI to patients in treatment for other SUD means missing opportunities to reduce health and economic costs while perpetuating a smoking culture.

Keywords: Smoking cessation; substance-related disorders; substance abuse treatment centers; attitude of health personnel; education.

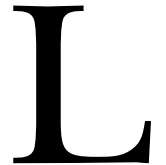
as tasas de tabaquismo en personas con trastorno por uso de sustancias (TUS) son de dos a cuatro veces mayores que las de la población general, alcanzando entre el $75 \%$ y el $85 \%$ (Guydish et al., 2016). Además, las personas con TUS fuman más cigarrillos al día y desarrollan niveles más altos de adicción a la nicotina que la población general, incluso en comparación con personas con otros trastornos psiquiátricos (Grant, Hasin, Chou, Stinson y Dawson, 2004). Por tanto, esta población tiene una muy elevada morbilidad y mortalidad relacionada con el tabaquismo ya que entre el $36 \%$ y el $49 \%$ de todas las muertes se atribuyen a afecciones relacionadas con el tabaquismo (Callaghan, Gatley, Sykes y Taylor, 2018). Sin embargo, las personas con TUS están interesadas en dejar de fumar cuando se les pregunta (Joseph, Nelson, Nugent y Willenbring, 2003; Nahvi, Richter, Li, Modali y Arnsten, 2006) y pueden lograr la abstinencia del tabaco a largo plazo (Smith, Mazure y McKee, 2014). De hecho, las intervenciones basadas en la evidencia dirigidas a los fumadores en tratamiento o recuperación del TUS aumentan la abstinencia del tabaco (Apollonio, Philipps y Bero, 2016), y dejar de fumar tiene un efecto positivo en los resultados del consumo de sustancias (McKelvey, Thrul y Ramo, 2017; Tsoh, Chi, Mertens y Weisner, 2011; Weinberger, Platt, Jiang y Goodwin, 2015).

Es más, las guías de práctica clínica animan a todos los médicos, incluidos los que trabajan en programas de tratamiento de TUS, a adoptar prácticas basadas en la evidencia para el tratamiento de la dependencia del tabaco (Camarelles et al., 2013; Fiore et al., 2008; National Institute for Health and Care Excellence, 2013; Rüther et al., 2014). De hecho, los centros de tratamiento ambulatorio de TUS son un entorno óptimo para las intervenciones para la cesación tabáquica (ICT), dado que la integración del tratamiento para la dependencia del tabaco ofrece un mensaje coherente dirigido a todas las dependencias de sustancias. Además, estas intervenciones están relacionadas con cambios positivos en el estilo de vida, con la reducción de los problemas de asistencia y con el logro de mejores tasas de abstinencia en comparación con los pacientes derivados a una clínica de cese externa (McFall et al., 2010).

Sin embargo, el tabaquismo entre las personas con TUS suele pasarse por alto, ya que cuatro de cada 10 profesiona- les de la salud mental (PSM) perciben barreras respecto de la implementación de ICT, tienen actitudes negativas hacia el abandono del tabaco y actitudes permisivas hacia el tabaquismo (Sheals, Tombor, McNeill y Shahab, 2016). Los fumadores también están sobrerrepresentados entre los PSM que trabajan en los servicios de tratamiento de TUS, lo que contribuye a normalizar el tabaquismo. Además, es menos probable que los fumadores impartan ICT (Pipe, Sorensen y Reid, 2009). Muchos de estos problemas contribuyen a perpetuar la cultura del tabaquismo entre los servicios de tratamiento de TUS (Bowman y Walsh, 2003).

Una revisión de la implementación de ICT en los centros de tratamiento de TUS en los Estados Unidos encontró que, aunque el $86 \%$ de los PSM preguntó a sus pacientes si fumaban, sólo se aconsejó al $40 \%$ de los pacientes que dejasen de fumar o se les evaluó para conocer su disposición para hacerlo (Knudsen, 2017). Sin embargo, se ha prestado poca atención a estos problemas en Europa, con escasos estudios realizados para evaluar la provisión de ICT en los centros de tratamiento de TUS. El objetivo de este estudio es examinar la adopción de ICT en los centros de tratamiento de TUS en Cataluña (España) y describir brevemente las ICT ofrecidas. También evaluamos las opiniones de sus responsables sobre la conveniencia y viabilidad de integrar las ICT en el contexto del tratamiento de otras adicciones. Por último, buscamos las variables asociadas con la impartición de ICT.

\section{Métodos}

\section{Diseño, muestra}

Los datos para este estudio transversal se recopilaron entre 2015 y 2016 mediante un cuestionario en línea. La población objetivo de la encuesta era gestores clínicos directamente responsables de los centros de tratamiento de TUS. Todos estos centros atendían únicamente a pacientes ambulatorios.

La Red de Atención a las Drogodependencias de Cataluña (CAS) está formada por 57 centros de tratamiento ambulatorio (excluidos los de los centros penitenciarios; $\mathrm{n}=2$ ). Cataluña es un territorio de más de 7,5 millones de habitantes en el noreste de España. Los CAS asisten a más de 16.000 pacientes por año. 


\section{Mediciones}

Diseñamos un cuestionario ad-hoc de 30 ítems para evaluar las ICT y las actitudes relacionadas con el abandono del hábito de fumar en el contexto de otras adicciones. El cuestionario se elaboró y consensuó entre los profesionales del Grupo de Trabajo de Tabaco en la Red de Atención a las Drogodependencias de Cataluña. El cuestionario (disponible en: shorturl.at/bnpv6) tenía cuatro partes que evaluaron diferentes áreas: 1) las características organizativas, 2) las características de la ICT, 3) la formación del personal en ICT y el interés en recibir formación nueva, y 4) las actitudes de los responsables respecto de la conveniencia y viabilidad de ofrecer ICT en sus centros. En la parte 1, 2 y 3 , todas las preguntas eran dicotómicas (sí/no), pedían números específicos (p. ej., el número de profesionales capacitados), o los encuestados podían seleccionar varias respuestas de una lista definida de opciones (p. ej., el tipo de ICT implementado). En la parte 4, las actitudes se evaluaron en una escala tipo Likert de 3 puntos, desde $1=d e$ acuerdo a 3 = en desacuerdo.

Además, los autores clasificaron los centros de tratamiento de TUS según tipo (con o sin salas de venopunción), ubicación en un entorno de atención sanitaria (p. ej., hospital, centro comunitario de salud mental o centro independiente), y población municipal (grande si $>50.000$ habitantes; pequeño si $<50.000$ habitantes).

\section{Procedimiento}

Se obtuvo un listado completo de los CAS del Departamento de Salud de la Generalitat de Catalunya $(n=57)$. A los responsables de cada centro se les envió por correo electrónico una carta de invitación en la que se explicaba el objetivo general de la encuesta, incluido el enlace a la encuesta. Los que no respondieron fueron invitados de nuevo por correo electrónico hasta tres veces. Tras los tres recordatorios, se solicitó su participación mediante llamada telefónica.

\section{Análisis de datos}

Se calcularon estadísticas descriptivas para tener una instantánea de las características organizativas de los centros participantes. Las características organizativas, la formación y las actitudes se trataron como variables independientes y la provisión de ICT como variable dependiente. Para comparar los centros con y sin ICT, se hicieron pruebas de Chi cuadrado para determinar las relaciones entre las variables categóricas, y pruebas $t$ de Student para las variables cuantitativas. Se calcularon las odds ratio(OR) con intervalos de confianza (IC) del $95 \%$ para las variables binarias. Se calculó la $d$ de Cohen para indicar el tamaño del efecto entre las medias de las variables cuantitativas. Las opiniones de los responsables se compararon con la prueba exacta de Fisher. Todas las pruebas fueron bilaterales y se realizaron con un alfa de
0,05. Todos los análisis estadísticos se realizaron con SPSS versión 20.0 para Windows.

\section{Resultados}

Se obtuvieron datos de 50 centros (tasa de respuesta del $87,7 \%$ ). Los que no respondieron no dieron razón específica respecto de su no participación. Entre los encuestados, los CAS eran muy diferentes en tamaño: el número promedio de profesionales (incluyendo al personal no clínico) fue 9,6 (intervalo de 2-34, $\mathrm{n}=479$ ). El personal sanitario representó el 89,8\% de todo el personal. En general, la profesión más común era psiquiatra $(26,9 \%)$, seguido por psicólogo $(20,9 \%)$, enfermera $(17,7 \%)$, trabajador social $(11,3 \%)$ y otros $(22,9 \%)$. Todos los centros contaban con al menos un psiquiatra y un psicólogo, ambos a tiempo parcial. Un centro no tenía enfermera y dos no tenían trabajador social. Un total de 16.859 pacientes nuevos fueron atendidos en todos los centros el año anterior (2014). El promedio de pacientes nuevos para cualquier TUS durante 2014 fue 344 (desviación típica 337,5; rango 0-1652).

El 46\% (n = 23) de los CAS implementaba algún tipo de ICT (ver Tabla 1 para una descripción). El $65 \%$ de ellos aceptaron pacientes sin otro TUS para cese del tabaquismo. Un total de 809 pacientes nuevos fueron tratados para dejar de fumar, que representan el 4,8\% de todos los pacientes nuevos en 2014.

Respecto a la formación, el 73,3\% (n = 315) de todos los profesionales no había recibido ningún tipo de formación en ICT. Además, el 66\% ( $\mathrm{n}=33)$ de los centros de tratamiento de TUS manifestó su interés en recibir formación.

En referencia a las actitudes de los responsables, el $78 \%$ estuvo de acuerdo con que «la ICT en los CAS complementa la prestación de atención primaria», y solo el $6 \%$ estuvo en desacuerdo; el $74 \%$ estuvo de acuerdo con que «las personas que quieren dejar de fumar tienen derecho a ser tratadas en los CAS» y solo el $2 \%$ estuvo en desacuerdo; el $74 \%$ estuvo de acuerdo con que «los profesionales que implementan ICT en los CAS necesitan mayor reconocimiento y el apoyo de las autoridades», mientras que el $8 \%$ estuvo en desacuerdo; el $67 \%$ estuvo de acuerdo con que «es necesario crear un protocolo común», mientras que el 12,5\% estuvo en desacuerdo; el $60 \%$ estuvo de acuerdo con que «todos los profesionales deberían estar capacitados en ICT», mientras que el 12\% estuvo en desacuerdo; y, por último, el $64 \%$ estuvo de acuerdo con que «todos los profesionales deben implementar ICT», mientras que el $10 \%$ estuvo en desacuerdo (el resto no estuvo de acuerdo ni en desacuerdo para cada afirmación).

Los centros que implementaban ICT atendieron a más pacientes que los que no implementaban ICT $(t=2,5 ; g l$ $=33 ; p=, 018)$, pero no se encontraron otras diferencias. En cuanto a la formación, el primero tenía más profesionales formados en ICT $(t=2,2 ; g l=47 ; p=, 034)$, aunque el interés por la formación no se asoció con la impartición 
Tabla 1. Principales características de las intervenciones para la cesación tabáquica implementadas en los Centros de Atención a las Drogodependencias (CAS) \%(n).

\begin{tabular}{|c|c|}
\hline \multicolumn{2}{|l|}{ Número de profesionales que implementan ICT } \\
\hline Médicos & $32,5(42)$ \\
\hline Psicólogos & $23,9(24)$ \\
\hline Enfermeras & $11,7(10)$ \\
\hline Trabajadores Sociales & $3,7(2)$ \\
\hline Otros & $1,1(1)$ \\
\hline Año de primera implementación de ICT, antes de 2000 & $52,2(12)$ \\
\hline Tiempo total dedicado a la implementación de ICT, menos del $10 \%$ del tiempo & $78,3(18)$ \\
\hline \multicolumn{2}{|l|}{ Tipo de intervención } \\
\hline Entrevista motivacional & $65,2(15)$ \\
\hline Psicoterapia individual & $65,2(15)$ \\
\hline Terapia grupal & $30,4(7)$ \\
\hline Uso de medicamentos para la deshabituación tabáquica & $87(20)$ \\
\hline Tiempo de seguimiento, 12 meses & $39,1(9)$ \\
\hline Número de visitas durante los primeros tres meses, 3-5 visitas & $52,2(12)$ \\
\hline \multicolumn{2}{|l|}{ Fumadores diana } \\
\hline Población general & $60,9(14)$ \\
\hline Población con enfermedades relacionadas con el tabaquismo & $60,9(14)$ \\
\hline Población con trastornos psiquiátricos & $65,2(15)$ \\
\hline \multicolumn{2}{|l|}{ Derivaciones } \\
\hline Autoderivaciones & $57,1(12)$ \\
\hline Atención primaria & $66,7(14)$ \\
\hline Médicos especialistas & $57,1(12)$ \\
\hline Centros de salud mental & $85,7(18)$ \\
\hline Salud laboral & $9,5(2)$ \\
\hline \multicolumn{2}{|l|}{ Lista de espera } \\
\hline Menos de 1 mes & $73,9(17)$ \\
\hline Lista de espera separada & $34,8(8)$ \\
\hline
\end{tabular}

de ICT. No se encontraron diferencias significativas en las opiniones de los responsables (Tabla 2).

\section{Discusión}

Según nuestro estudio, casi la mitad de los CAS en Cataluña implementaba ICT. Los centros que implementaban ICT atendieron a más pacientes que los que no implementaban ICT. La mayoría de ellos atendieron a pacientes sin TUS aparte del tabaquismo. La ICT representó menos del $5 \%$ de las intervenciones en pacientes nuevos. La mayoría de los centros informó de la prescripción de medicamentos para la deshabituación tabáquica, realizaron intervenciones basadas en la entrevista motivacional y psicoterapia individual; sin embargo, era poco común que tuviesen grupos de cese del tabaquismo. Además, solo uno de cada cuatro profesionales había recibido formación, y era más probable que los centros con profesionales formados implementasen ICT. La mayoría de los responsables estuvo de acuerdo en que todos los profesionales deberían implementar ICT.

Hay solo dos estudios anteriores sobre el tema realizados en España. Becoña et al. (2006) encontraron que el $55,6 \%$ de los centros de tratamiento de TUS (con una tasa de respuesta del 53\%) de 11 de las 17 comunidades au- tónomas de España (incluida Cataluña) implementaron ICT en 2004. Este resultado es bastante similar al hallado en nuestro estudio ( $55,6 \%$ vs. $46 \%$ en nuestro estudio), aunque nuestros resultados se obtuvieron 10 años después. Además, en estos años se han producido cambios importantes en este campo, ya que en 2011 entró en vigor la prohibición total de fumar en todos los servicios sanitarios (incluidos los servicios de tratamiento psiquiátrico hospitalario y servicios de tratamiento de TUS ambulatorio). Recientemente, en Cataluña se han producido mejoras en el control del tabaco como el aumento de la sensibilización de los profesionales sanitarios sobre los riesgos del tabaquismo, la formación en cesación tabáquica y el buen cumplimiento de la prohibición nacional de fumar (Ballbè, Gual, Nieva, Saltó y Fernández, 2016). Sin embargo, estas mejoras no parecen haber cambiado las prácticas de los CAS. El otro estudio realizado en Asturias (otra comunidad autónoma), encontró que solo el $20 \%$ de los centros de tratamiento de TUS implementaron ICT (González-Roz et al., 2019). Las disparidades pueden reflejar una implementación desigual de ICT en España.

Según nuestro estudio, pertenecer a un centro hospitalario no se asoció con la implementación de ICT, a diferencia de algunos estudios realizados en los EE.UU. (Eby y Laschober, 2013; Eby, Laschober y Muilenburg, 2015). Las 
Tabla 2. Características organizativas, formación del personal y opiniones de los responsables en los CAS en cuanto a la implementación o no de ICT $(n=50)$.

\begin{tabular}{|c|c|c|c|c|}
\hline & $\begin{array}{l}\text { Centros de TUS que } \\
\text { implementan ICT } \\
(n=23) \\
\% \text { o media (SD) }\end{array}$ & $\begin{array}{c}\text { Centros de TUS que } \\
\text { NO implementan ICT } \\
(n=27) \\
\% \text { o media (SD) }\end{array}$ & $\begin{array}{l}d^{1} \text { de } \\
\text { Cohen }\end{array}$ & RM (IC 95\%) $)^{2}$ \\
\hline \multicolumn{5}{|l|}{ Número de profesionales } \\
\hline Médicos & $2,96(2,36)$ & $2,26(1,38)$ & & \\
\hline Psicólogos & $2,3(1,29)$ & $1,76(0,75)$ & & \\
\hline Enfermeras & $1,76(0,94)$ & $1,67(1,04)$ & & \\
\hline Trabajadores Sociales & $1,08(0,56)$ & $1,09(0,39)$ & & \\
\hline Otros & $2,15(3,76)$ & $2,22(3,4)$ & & \\
\hline Todos & $10,3(6,7)$ & $9(5,84)$ & & \\
\hline Pacientes nuevos en 2014 & $468,48(402,61)$ & $234(221,87)$ & 0,72 & \\
\hline Municipio grande (>50 000 habitantes) & $69,6 \%$ & $51,9 \%$ & & $2.1(0,66-6,8)$ \\
\hline Centros con salas de venopunción & $8,7 \%$ & $25,9 \%$ & & $0,27(0,05-1,47)$ \\
\hline Ubicado en un entorno sanitario & $43,5 \%$ & $25,9 \%$ & & $2,2(0,67-7,2)$ \\
\hline $\begin{array}{l}\text { Número de profesionales interesados en recibir formación } \\
\text { en cese de tabaquismo }\end{array}$ & $56,5 \%$ & $74,1 \%$ & & $0,45(0,14-1,5)$ \\
\hline \multicolumn{5}{|l|}{ Actitudes de los responsables } \\
\hline $\begin{array}{l}\text { (1) ICT implementado en CAS complementa } \\
\text { los servicios de atención primaria, de acuerdo }{ }^{3} \text {. }\end{array}$ & $78,3 \%$ & $77,8 \%$ & & \\
\hline $\begin{array}{l}\text { (2) Las personas que desean dejar de fumar tienen el derecho a recibir } \\
\text { tratamiento en los CAS, de acuerdo. }\end{array}$ & $69,6 \%$ & $77,8 \%$ & & \\
\hline $\begin{array}{l}\text { (3) Los profesionales que implementan ICT necesitan mayor } \\
\text { reconocimiento público y apoyo de las autoridades sanitarias, de acuerdo. }\end{array}$ & $73,9 \%$ & $74,1 \%$ & & \\
\hline (4) Es necesario crear un protocolo común sobre ICT, de acuerdo. & $52,2 \%$ & $80 \%$ & & \\
\hline $\begin{array}{l}\text { (5) Todos los profesionales sanitarios deben recibir formación } \\
\text { en el cese del tabaquismo, de acuerdo. }\end{array}$ & $52,2 \%$ & $66,7 \%$ & & \\
\hline
\end{tabular}

Nota. ${ }^{1}$ Se calculó el $d$ de Cohen para las variables continuas. ${ }^{2} \mathrm{OR}=$ Odds ratio; IC = intervalo de confianza; calculado para las variables dicotómicas.

${ }_{3}^{3}$ Porcentaje de aquellos que están de acuerdo.

diferencias organizativas pueden explicar estos resultados. Otras variables asociadas a las ICT, como organización sin ánimo de lucro, reembolso de los tratamientos o financiación (Eby et al., 2015), no pudieron analizarse porque todos los centros encuestados eran públicos y en el momento del estudio la sanidad pública en Cataluña no cubría los medicamentos para la deshabituación tabáquica.

En cuanto a la formación, nuestros resultados son consistentes con otros estudios que concluyen que es más probable que los profesionales formados tienen más probabilidades de impartir ICT (Carson et al., 2012), y que la formación es escasa y una necesidad pendiente en estos centros (Knudsen, 2017).

Las actitudes de los responsables no diferían según la implementación o no de ICT. Otros estudios encontraron una actitud de mayor apoyo de las ICT por parte del personal que trabaja en centros que implementan ICT, también en centros que atienden a mujeres embarazadas, pero de menor apoyo cuando los centros ofrecían tratamiento residencial (Fuller et al., 2007). Sin embargo, en nuestro estudio los centros fueron más homogéneos, ya que todos los centros aceptaban a todas las poblaciones y ninguno ofrecía tratamiento residencial. Por tanto, no se pudo analizar el papel de esas variables.

Este estudio aborda un tema escasamente investigado en Europa, y además es el primero en describir las ICT en los CAS en Cataluña. Una alta tasa de respuesta $(87,7 \%)$ es una fortaleza. Este estudio también aclara las opiniones de los responsables sobre la implementación de ICT en centros de tratamiento de TUS por, según el conocimiento de los autores, primera vez en España. Este estudio también tiene limitaciones. Como estudio transversal, no se proporciona información sobre cambios en la adopción de ICT a lo largo del tiempo. Además, los responsables proporcionan la información y no se pudo verificar la precisión de las respuestas autoinformadas. Por último, aunque se obtuvo una alta tasa de respuesta, el tamaño muestral fue bajo y puede haber afectado los resultados.

La investigación futura debe centrarse en las barreras a la implementación de ICT en los centros de tratamiento de TUS. Dado que la formación parece ser un tema clave, se debe proporcionar e incentivar la educación y la formación 
continua para los profesionales que trabajan en los centros de tratamiento de TUS. Además, un estudio cualitativo de las actitudes del personal mejoraría la comprensión de este asunto. Asimismo, dado que algunos medicamentos basados en la evidencia para la deshabituación tabáquica están financiados desde principios de 2020 por el Ministerio de Salud Pública español, se justifican nuevos estudios.

Este estudio presenta una gran necesidad clínica pendiente. Las conclusiones deberían ser una llamada de atención para las autoridades sanitarias. Implementar ICT no sistemáticamente en pacientes en tratamiento por otros TUS significa perder oportunidades para reducir los costes del consumo de tabaco y otras drogas, a la vez que perpetuar una cultura fumadora.

\section{Reconocimientos}

Los autores quieren agradecer a todos los responsables directos de los centros de la Red de Atención de las Drogodependencias de Cataluña su participación en el estudio. Todos los autores declaran que no han recibido apoyo económico alguno para este estudio.

\section{Conflicto de intereses}

Los autores declaran la inexistencia de conflicto de interés.

\section{Referencias}

Apollonio, D., Philipps, R. y Bero, L. (2016). Interventions for tobacco use cessation in people in treatment for or recovery from substance abuse. The Cochrane Library, 12, 1-10. doi:10.1002/14651858.CD010274.

Becoña, E., García, V., de Echave, J., Feránadez, J. J., Sánchez, M. del M., Terán, A.,... Castillo, A. (2006). Tratamiento del tabaquismo en los centros de drogodependencias y alcoholismo en España. Estado de la situación y perspectivas futuras. Adicciones, 18, 23-38. doi:10.20882/ adicciones.353.

Ballbè, M., Gual, A., Nieva, G., Saltó, E. y Fernández, E. (2016). Deconstructing myths, building alliances: A networking model to enhance tobacco control in hospital mental health settings. Gaceta Sanitaria, 30, 389-392. doi:10.1016/j.gaceta.2016.04.017.

Bowman, J. A. y Walsh, R. A. (2003). Smoking intervention within alcohol and other drug treatment services: A selective review with suggestions for practical management. Drug and Alcohol Review, 22, 73-82. doi:10.1080/09 59523021000059857.

Callaghan, R. C., Gatley, J. M., Sykes, J. y Taylor, L. (2018). The prominence of smoking-related mortality among individuals with alcohol- or drug-use disorders. Drug and Alcohol Review, 37, 97-105. doi:10.1111/dar.12475.
Camarelles, F., Dalmau, R., Clemente, L., Díaz Maroto, J. L., Lozano, A., Pinet, M. C. y Grupo colaborador CNPT. (2013). Documento de consenso para la atención clínica al tabaquismo en España. Medicina Clinica, 140, 272e1-272e12. doi:10.1016/j.medcli.2012.10.013.

Carson, K., Verbiest, M., Crone, M., Brinn, M., Esterman, A., Assendelft, W. y Smith, B. (2012). Training health professionals in smoking cessation. Cochrane Database of Systematic Reviews, 5. doi:10.1002/14651858.

Eby, L. T. de T. y Laschober, T. C. (2013). Perceived implementation of the Office of Alcoholism and Substance Abuse Services (OASAS) tobacco-free regulation in NY State and clinical practice behaviors to support tobacco cessation: A repeated cross-sectional study. Journal of Substance Abuse Treatment, 45, 83-90. doi:10.1016/j. jsat.2013.01.001.

Eby, L. T., Laschober, T. C. y Muilenburg, J. L. (2015). Sustained, new, never, and discontinued tobacco cessation services adopters. Journal of Substance Abuse Treatment, 49, 8-14. doi:10.1016/j.jsat.2014.07.017.

Fiore, M. C., Jaén, C. R., Baker, T. B., Bailey, W. C., Benowitz, N., Curry, S. J.,... Wewers, M. E. (2008). Treating Tobacco Use and Dependence: 2008 Update. Quick Reference Guide for Clinicians. Rockville, MD: US Department of Health and Human Services, Public Health Service.

Fuller, B. E., Guydish, J., Tsoh, J., Reid, M. S., Resnick, M., Zammarelli, L.,... McCarty, D. (2007). Attitudes toward the integration of smoking cessation treatment into drug abuse clinics. Journal of Substance Abuse Treatment, 32, 53-60. doi:10.1016/j.jsat.2006.06.011.

González-Roz, A., Ruano, L., Aonso-Diego, G., García-Pérez, Á., Weidberg, S. y Secades-Villa, R. (2019). Abordaje del tabaquismo en centros de tratamiento de drogodependencias: Implicaciones clínicas y recomendaciones para su implementación. Adicciones, 31, 327-329. doi:10.20882/adicciones.1270.

Grant, B. F., Hasin, D. S., Chou, S. P., Stinson, F. S. y Dawson, D. A. (2004). Nicotine dependence and psychiatric disorders in the United States. Archives of General Psychiatry, 61, 1107-1115. doi:10.1001/archpsyc.61.11.1107.

Guydish, J., Passalacqua, E., Pagano, A., Martínez, C., Le, T., Chun, J.,... Delucchi, K. (2016). An international systematic review of smoking prevalence in addiction treatment. Addiction (Abingdon, England), 111, 220-230. doi:10.1111/add.13099.

Joseph, A. M., Nelson, D. B., Nugent, S. M. y Willenbring, M. L. (2003). Timing of alcohol and smoking cessation (TASC): Smoking among substance use patients screened and enrolled in a clinical trial. Journal of Addictive Diseases, 22, 87-107. doi:10.1300/J069v22n04_08.

Knudsen, H. K. (2017). Implementation of smoking cessation treatment in substance use disorder treatment settings: A review. The American Journal of Drug and Alcohol Abuse, 43, 215-225. doi:10.1080/00952990.2016.1183019. 
McFall, M., Saxon, A. J., Malte, C. A., Chow, B., Bailey, S., Baker, D. G.,... Lavori, P. W. (2010). Integrating tobacco cessation into mental health care for posttraumatic stress disorder: A randomized controlled trial. JAMA, 304, 2485-2493. doi:10.1001/jama.2010.1769.

McKelvey, K., Thrul, J. y Ramo, D. (2017). Impact of quitting smoking and smoking cessation treatment on substance use outcomes: An updated and narrative review. Addictive Behaviors, 65, 161-170. doi:10.1016/j.addbeh.2016.10.012.

Nahvi, S., Richter, K., Li, X., Modali, L. y Arnsten, J. (2006). Cigarette smoking and interest in quitting in methadone maintenance patients. Addictive Behaviors, 31, 21272134. doi:10.1016/j.addbeh.2006.01.006.

National Institute for Health and Care Excellence. (2013). Smoking: Acute, maternity and mental health services. Public health guideline [PH48]. Recuperado de www.nice. org.uk/guidance/ph48/evidence.

Pipe, A., Sorensen, M. y Reid, R. (2009). Physician smoking status, attitudes toward smoking, and cessation advice to patients: An international survey. Patient Education and Counseling, 74, 118-123. doi:10.1016/j.pec.2008.07.042.

Rüther, T., Bobes, J., De Hert, M., Svensson, T. H., Mann, K., Batra, A.,1... Möller, H. J. (2014). EPA Guidance on tobacco dependence and strategies for smoking cessation in people with mental illness. European Psychiatry: The Journal of the Association of European Psychiatrists, 29, 65-82. doi:10.1016/j.eurpsy.2013.11.002.

Sheals, K., Tombor, I., McNeill, A. y Shahab, L. (2016). A mixed-method systematic review and meta-analysis of mental health professionals' attitudes toward smoking and smoking cessation among people with mental illnesses. Addiction, 111, 1536-1553. doi:10.1111/add.13387.

Smith, P. H., Mazure, C. M. y McKee, S. A. (2014). Smoking and mental illness in the U.S. population. Tobacco Control, 23, e147-53. doi:10.1136/tobaccocontrol-2013-051466.

Tsoh, J. Y., Chi, F. W., Mertens, J. R. y Weisner, C. M. (2011). Stopping smoking during first year of substance use treatment predicted 9-year alcohol and drug treatment outcomes. Drug and Alcohol Dependence, 114, 110-118. doi:10.1016/j.drugalcdep.2010.09.008.

Weinberger, A. H., Platt, J., Jiang, B. y Goodwin, R. D. (2015). Cigarette smoking and risk of alcohol use relapse among adults in recovery from alcohol use disorders. Alcoholism: Clinical and Experimental Research, 39, 19891996. doi:10.1111/acer.12840. 
